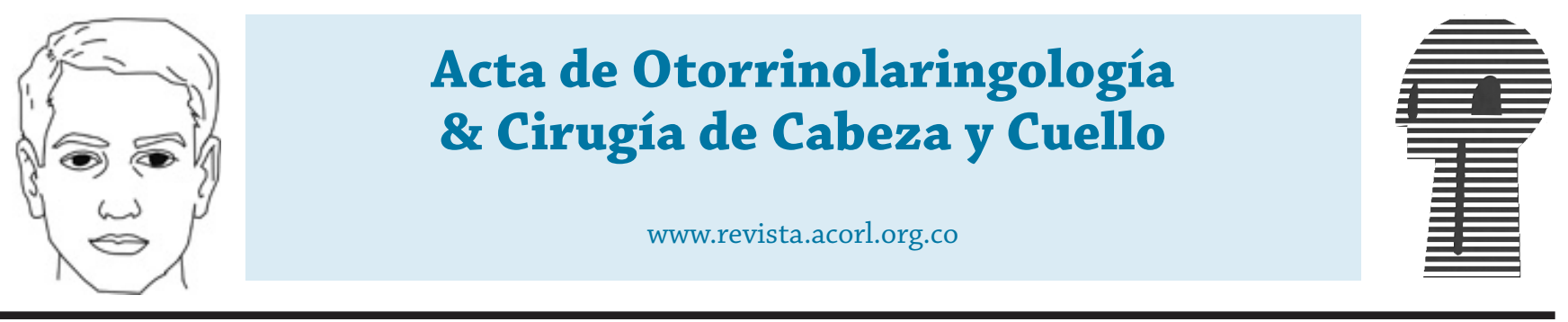

Trabajos originales

\title{
Análisis clínico y terapéutico ante la patología obstructiva crónica de las glándulas salivales
}

\section{Clinical and therapeutic analysis to the chronic obstructive pathology of the salivary glands}

\author{
Esteban Reig-Montaner*, María Antón-Almero*, María José Ferrer-Ramírez**, Iván Doménech-Máñez*, \\ Natalia Gordillo-Gayo*, Arantxa Torres-Roselló***, Marta Faubel-Serra***. \\ * Servicio de Otorrinolaringología y Cirugía de Cabeza y Cuello, Hospital General Universitario de Castellón. Castelló de la \\ Plana, Castelló. \\ ** Servicio de Otorrinolaringología y Cirugía de Cabeza y Cuello, Hospital General Universitario de Castellón. Castelló de la \\ Plana, Castelló. Universidad Jaime I. Facultad de Ciencias de la Salud. Castelló de la Plana, Castelló. \\ *** Universidad Jaime I. Facultad de Ciencias de la Salud. Castelló de la Plana, Castelló.
}

Forma de citar: Reig-Montaner E, Antón-Almero M, Ferrer-Ramírez MJ, Doménech-Máñez I, Gordillo-Gayo N, Torres-Roselló A, Faubel-Serra M. Análisis clínico y terapéutico ante la patología obstructiva crónica de las glándulas salivales. Acta otorrinolaringol. cir. cabeza cuello. 2021;49(4): 279-284. DOI.10.37076/acorl.v49i4.610

\section{INFORMACIÓN DEL ARTÍCULO}

Historia del artículo:

Recibido: 7 de abril de 2021

Evaluado: 22 de noviembre de 2021

Aceptado: 9 de diciembre de 2021

Palabras clave (DeCS):

endoscopia, litiasis, glándula submandibular, glándula parótida.

\section{RESUMEN}

Introducción: gran parte de la población sufre procesos relacionados con las glándulas salivales, que, con los avances técnicos, se tiende cada vez más a tratar de una manera mínimamente invasiva. Objetivos: remarcar las indicaciones y las diferencias entre los abordajes comunes y los mínimamente invasivos, guiados por el sialoendoscopio. Además, describir la presentación clínica y el estudio de dichos pacientes. Diseño: realizamos un estudio descriptivo, observacional, longitudinal y retrospectivo sobre un grupo de 67 pacientes diagnosticados con patología obstructiva crónica no tumoral de las glándulas. Material y métodos: revisamos los datos referentes a la edad, sexo, hábitos tóxicos, enfermedades sistémicas o autoinmunes asociadas, radioterapia o tratamiento con yodo radiactivo $\left(\mathrm{I}^{131}\right)$, síntomas asociados y resultados del examen físico y radiológico efectuados, así como el tratamiento efectuado. En mayo de 2019 incorporamos la técnica de sialoendoscopia al manejo de esta patología. Resultados: desde la incorporación de la sialoendoscopia, los casos de patología litiásica a nivel del tercio distal del conducto de Wharton se abordaron mediante exéresis de la litiasis sobre el suelo de la boca con ayuda del sialoen-

Correspondencia:

Esteban Reig-Montaner

Email: esteban.reig17@gmail.com

Dirección: Ciudad de Barcelona, 29. Gandía (46702), Valencia. 
Key words (MeSH):

Endoscopy; Lithiasis; Submandibular Gland; Parotid Gland. doscopio. Realizamos una sialoendoscopia diagnóstico-terapéutica en pacientes con clínica obstructiva crónica no litiásica. Discusión: el abordaje mínimamente invasivo permite una recuperación más temprana con una adecuada función glandular tras la cirugía. No solo es útil en la patología litiásica, sino que también presenta buenos resultados en patología autoinmune. Conclusión: las técnicas mínimamente invasivas han hecho que el manejo haya cambiado, limitando la realización de resecciones glandulares.

\section{ABSTRACT}

Introduction: A large part of the population suffers from processes related to the salivary glands, which with new advances in technology tends to be treated in a minimally invasive way. Goals: To highlight the indications and differences between common and minimally invasive approaches, guided by the sialoendoscope. In addition, to describe the clinical presentation and the study of these patients. Design: We carried out a descriptive, observational, longitudinal and retrospective study on a group of 67 patients diagnosed with non-tumorous chronic obstructive pathology of the glands. Material and methods: We review the data regarding age, sex, toxic habits, associated systemic or autoimmune diseases, radiotherapy or treatment with ${ }^{131}$ (radioactive iodine), associated symptoms and results of the physical and radiological examination carried out. As well as the given treatment. In May 2019 we incorporated the sialoendoscopy to the management of this pathology. Results: Since the incorporation of sialoendoscopy, cases of lithiasic pathology at the distal $1 / 3$ of Wharton's duct were approached by excision of the stone on the floor of the mouth using sialoendoscopy. We perform diagnostic-therapeutic sialoendoscopy in patients with non-lithiasic chronic obstructive symptoms. Discussion: The minimally invasive approach allows an earlier recovery with adequate glandular function after surgery. It is not only useful in lithiasic pathology, but it also has good results in autoimmune pathology. Conclusion: Minimally invasive techniques have changed management, limiting the neck open surgeries.

\section{Introducción}

La patología obstructiva crónica de las glándulas salivales es un problema de salud importante que afecta al 1,2\% de la población, siendo el $66 \%$ de los casos de etiología benigna (1). En los últimos años, el tratamiento de las patologías está evolucionando rápidamente hacia las técnicas mínimamente invasivas, un enfoque basado en la medicina personalizada y los tratamientos específicos. En el caso de los trastornos de las glándulas salivales, las mejoras tecnológicas como la miniaturización de los sistemas videoendoscópicos, los avances en radiología intervencionista y el desarrollo de nuevas estrategias farmacológicas permiten ahora un manejo personalizado de la sialoadenitis recurrente obstructiva. El aumento del conocimiento de los mecanismos obstructivos ha favorecido enfoques conservadores que dejan una glándula funcional en aproximadamente el $97 \%$ de los pacientes (2). El papel clave de la sialoendoscopia en el manejo de la sialoadenitis obstructiva ha sido destacado en la literatura internacional (2-5).

El objetivo de este trabajo es realizar un estudio descriptivo de una población de pacientes con patología obstructiva de las glándulas salivales e identificar las características de la población, la rentabilidad diagnóstica de las diferentes técnicas de imagen y el abordaje de esta patología.

\section{Material y métodos}

Se realizó un estudio descriptivo, observacional, longitudinal y retrospectivo sobre un grupo de 67 pacientes diagnosticados con patología obstructiva crónica no tumoral de las glándulas salivales. El período analizado es el comprendido entre enero de 2017 y noviembre de 2020.

Los pacientes estudiados fueron remitidos al servicio de otorrinolaringología desde los servicios de urgencias, reumatología, endocrinología y atención primaria.

Se consideró como susceptibles de padecer patología no tumoral obstructiva crónica salival a aquellos pacientes con clínica salival de dolor, tumefacción y aumento de tamaño de una o más glándulas salivales (glándulas submaxilar y parótida) con más de tres episodios en los últimos seis meses, con o sin resolución espontánea. Se excluyeron aquellos pacientes diagnosticados de patología tumoral o que hayan presentado patología salival infecciosa, o menos de dos episodios de clínica salival obstructiva en los últimos seis meses resueltos espontáneamente. 
En todos los casos se procedió a completar el diagnóstico con las técnicas de imagen disponibles. Se realizó una ecografía de glándulas salivales por parte del servicio de radiología a casi todos los pacientes, ya que es una técnica de bajo coste y no invasiva, con una elevada sensibilidad y especificidad. Cuando se observó litiasis en la ecografía o bien se palpó en el trayecto del ducto, se realizó una tomografía computarizada (TC), técnica con alta sensibilidad para detectar litiasis radiopacas, determinar número, dimensión y localización de las mismas y permite diferenciar un cálculo voluminoso de un conglomerado de cálculos pequeños (6). La sialorresonancia (sialo-RM) se realizó en pacientes con patología parotídea; esta consiste en utilizar la propia saliva del paciente como contraste y permite la visualización completa de los conductos de las glándulas y el parénquima.

Se revisan los datos referentes a la edad, sexo, hábitos tóxicos, enfermedades sistémicas o autoinmunes asociadas, radioterapia o tratamiento con I131, síntomas asociados y resultados del examen físico y radiológico efectuados.

En mayo de 2019 se incorporó la sialoendoscopia al manejo de esta patología. Esta técnica permite la exploración del conducto salival con el fin de detectar cualquier anormalidad en los ductos. En el mismo acto se pueden tratar aquellas alteraciones que se pueden resolver de manera intraductal, utilizando la propia óptica y su canal de trabajo.

La recogida de datos se realizó a través de MS Excel y el análisis estadístico, así como la elaboración de gráficos y tablas mediante el paquete estadístico IBM ${ }^{\circledR}$ SPSS ${ }^{\circledR}$ Statistics.

Se realizó la estadística descriptiva de las variables cualitativas y cuantitativas para, a continuación, hacer la estadística bivariada que permita relacionar las variables que son objeto de estudio.

El análisis descriptivo de las variables se realizó mediante frecuencias y porcentajes para las cualitativas, y mediante medidas de tendencia central (media aritmética) y de dispersión (desviación estándar [DE]) para las cuantitativas.

El estudio fue aprobado por el Comité de ética de Investigación Clínica.

\section{Resultados}

De los 67 pacientes con patología obstructiva crónica no tumoral, el 65,7 \% (44 pacientes) eran mujeres, siendo la edad media de $54 \pm 17$ años. La mayoría de los pacientes fueron remitidos desde atención primaria $(65,7 \%)$ y urgencias $(19,4$ $\%$ ), aunque también se derivaron siete pacientes de reumatología, dos de endocrinología y tres de pediatría.

El antecedente médico más prevalente fue la diabetes mellitus (DM) tipo 1 (17,9\%), junto con el síndrome seco secundario $(17,9 \%)$. Cuatro pacientes padecían padeciendo hipotiroidismo, tres de artritis reumatoide, uno de lupus eritematoso sistémico y cuatro pacientes con otras enfermedades autoinmunes. Lo más frecuente fueron los pacientes sanos sin antecedentes médicos (71,64 \%) (Tabla 1).

\section{Tabla 1. Antecedentes personales}

\begin{tabular}{|l|l|}
\hline Pacientes sanos & $48(71,64 \%)$ \\
\hline DM tipo I & $12(17,9 \%)$ \\
\hline Malnutrición & $1(1,5 \%)$ \\
\hline Síndrome de Sjögren & $2(3 \%)$ \\
\hline Síndrome seco secundario & $12(17,9 \%)$ \\
\hline I131 & $3(4,5 \%)$ \\
\hline
\end{tabular}

Elaboración propia de los autores.

El 73,1 \% de los pacientes referían clínica de más de un año de evolución, presentando en su mayoría patología uniglandular $(88,1 \%)$, siendo la glándula submaxilar la más afectada $(68,7 \%)$.

En cuanto a la presentación clínica, la mayoría referían un aumento en el tamaño de la glándula $(64,2 \%)$, seguido de dolor $(58,2 \%)$. En 17 pacientes $(25,4 \%)$ se palpó un cálculo en la exploración; el 47,01\% en el tercio proximal, mientras que en el 52,99\% se encontró en los dos tercios distales (Tabla 2).

Tabla 2. Presentación clínica
\begin{tabular}{|l|l|}
\hline Xerostomía & $10(14,9 \%)$ \\
\hline Dolor & $39(58,2 \%)$ \\
\hline Masa & $43(64,2 \%)$ \\
\hline Eritema de papila & $9(13,4 \%)$ \\
\hline Saliva purulenta & $8(11,9 \%)$ \\
\hline Cálculo palpable & $\mathbf{N}=17(25,4 \%)$ \\
\hline $1 / 3$ proximal & $8(47,01 \%)$ \\
\hline $2 / 3$ distal & $9(52,99 \%)$ \\
\hline
\end{tabular}

Elaboración propia de los autores.

Los resultados de las diferentes pruebas de radiodiagnóstico se recogen en la Tabla 3. La prueba más realizada fue la ecografía $(91,04 \%)$, siendo el hallazgo más prevalente la inflamación crónica $(63,93 \%)$ seguido de la litiasis $(36,06$ $\%$ ). En los pacientes en los que se sospechaba la presencia de una patología litiásica o de la glándula submaxilar, se realizó TC $(38,81 \%)$. Por el contrario, si se sospechaba de patología inflamatoria, estenótica o de la glándula parótida, se realizó sialo-RM (19,4 \%).

La patología litiásica fue más frecuente en la glándula submaxilar frente a la parótida, con una $\mathrm{p}=0,004$. La patología no litiásica supuso un $47,76 \%$ de la muestra (32 pacientes), mientras que la litiásica supuso un 52,24 \% (35 pacientes), siendo estas en un $82,86 \%$ de los casos litiasis submaxilares, y en un 17,14 \% parotídeas (Figura 1).

El tamaño de la litiasis observado se clasificó en rangos de $<4 \mathrm{~mm}$, de $4 \mathrm{~mm}-8 \mathrm{~mm}$ y de $>8 \mathrm{~mm}$. El $47,85 \%$ de las litiasis fueron de $4 \mathrm{~mm}-8 \mathrm{~mm}$ de tamaño, el cual fue el rango de tamaño más frecuente.

Los pacientes fueron tratados mediante tratamiento médico, cirugía abierta, cirugía local transoral en consulta, 
Tabla 3. Hallazgos radiológicos

\begin{tabular}{|l|l|}
\hline Ecografía & $\mathbf{N}=61(91,04 \%)$ \\
\hline Litiasis & $22(36,06 \%)$ \\
\hline Estenosis & $2(3,27 \%)$ \\
\hline Inflamación crónica & $39(63,93 \%)$ \\
\hline Dilatación & $17(27,87 \%)$ \\
\hline TC & $\mathbf{N}=26(38,81 \%)$ \\
\hline Litiasis & $19(73,08 \%)$ \\
\hline Estenosis & $1(3,85 \%)$ \\
\hline Inflamación crónica & $3(11,54 \%)$ \\
\hline Dilatación & $7(26,92 \%)$ \\
\hline Sialo-RM & $\mathbf{N}=13(19,4 \%)$ \\
\hline Litiasis & $1(7,69 \%)$ \\
\hline Estenosis & $3(23,08 \%)$ \\
\hline Inflamación crónica & $9(69,23 \%)$ \\
\hline Dilatación & $9(69,23 \%)$ \\
\hline
\end{tabular}

Elaboración propia de los autores.

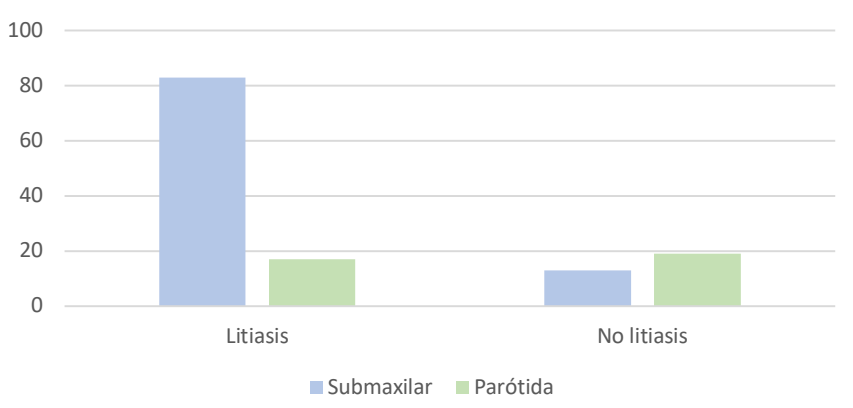

Figura 1. Distribución según la glándula de la patología litiásica y no litiásica. Elaboración propia de los autores.

sialoendoscopia básica y sialoendoscopia combinada (cirugía transoral con ayuda de sialoendoscopio). Cada una de estas técnicas se realizaba teniendo en cuenta la causa de la patología, el tamaño de la litiasis y las preferencias del paciente (Tabla 4).

\begin{tabular}{|l|l|}
\hline Tabla 4. Tratamiento & $8(11,9 \%)$ \\
\hline Cirugía abierta & $4(6 \%)$ \\
\hline Cirugía local transoral en consulta & $7(10,44 \%)$ \\
\hline Sialoendoscopia básica & $10(14,9 \%)$ \\
\hline Sialoendoscopia combinada &
\end{tabular}

Elaboración propia de los autores.

La mayoría de los pacientes de nuestra muestra recibió tratamiento médico y control de evolución. En el caso de los pacientes a los que se diagnosticó una litiasis en el 1/3 distal del conducto, próximo a la papila, se realizó exéresis de la litiasis en consultas externas bajo anestesia local (cuatro pacientes).

Previo a la incorporación de la técnica de sialoendoscopia (mayo de 2019), y en aquellos casos de patología litiásica a nivel de $1 / 3$ proximal del conducto de Wharton, se realizó submaxilectomía (ocho pacientes). Desde la incorporación de la sialoendoscopia, estos mismos casos se abordaron mediante exéresis de la litiasis (tamaño $>7-8 \mathrm{~mm}$ ) sobre el suelo de la boca con ayuda del sialoendoscopio (10 pacientes). Únicamente se realizó submaxilectomía en una paciente con una litiasis intraglandular de $1,5 \mathrm{~cm}$ de tamaño.

Cuatro pacientes con litiasis $<4 \mathrm{~mm}$ a nivel submaxilar están a la espera de la realización de sialoendoscopia básica y extracción del cálculo.

Se realizó una sialoendoscopia diagnóstico-terapéutica en siete pacientes: cinco casos de parotiditis crónica (tres con síndrome de Sjögren, uno con síndrome seco secundario y uno sin enfermedad asociada) y dos casos de submaxilitis crónica (una secundaria a lupus eritematoso sistémico y uno sin enfermedad asociada). En estos casos los hallazgos durante la sialoendoscopia fueron de palidez mucosa, ausencia de vascularización, depósitos fibrinosos, tapones mucosos y zonas de estenosis. Tras la exploración y lavado de los conductos, se instiló $80 \mathrm{mg}$ de metilprednisolona. Dos pacientes (uno con antecedente de tratamiento con I131 y uno sin enfermedad asociada) están a la espera de una sialoendoscopia diagnóstico-terapéutica.

\section{Discusión}

La enfermedad obstructiva de las glándulas salivales es una patología muy frecuente y puede ser causada por litiasis, estenosis ductal, tapones fibromucinosos o variantes anatómicas del sistema ductal. La glándula submaxilar está involucrada en el $80 \%-90 \%$ de los casos, seguida de la parótida (5\%-10\%) y sublingual $(<1 \%)$ (7). Tradicionalmente su manejo implica medidas conservadoras para aumentar el flujo saliva. Sin embargo, los casos refractarios pueden requerir de un tratamiento quirúrgico (8).

La patología obstructiva de las glándulas salivales afecta con mayor frecuencia más a las mujeres que a los hombres, hecho que se refleja en nuestra serie, con un porcentaje para las mujeres de $65,7 \%$ de los casos. En este estudio, la edad media fue de $54 \pm 17$ años, lo que coincide con la literatura, siendo el rango de edades más frecuente en torno a los 50-60 años $(9,10)$.

Existen trastornos de la secreción salival secundarios al uso de medicamentos (sobre todo los neuropsicótropos), enfermedad de Sjögren y otras patologías autoinmunes y la radioterapia de cabeza y cuello. En nuestro estudio, la mayoría de los pacientes no tomaban medicación que pudiera favorecer la presentación de una patología obstructiva crónica salival. El 34,69 \% de los pacientes de nuestra serie no presentaba patologías concomitantes, mientras que el 20,9\% padecía una enfermedad autoinmune.

Se referencia ampliamente en la literatura que la causa más frecuente de obstrucción es la litiasis a nivel del conducto salival (>50\%). Aproximadamente el $80 \%$ de estas litiasis van a afectar al conducto de Wharton, mientras que un $20 \%$ se 
producirán en el de Stenon. La segunda causa más frecuente de sialoadenitis obstructiva es la estenosis ductal, que ocurre más frecuentemente en la glándula parótida (11).

Se refiere que aproximadamente el $50 \%$ de las litiasis submaxilares se encuentran en el tercio distal del conducto, el $20 \%$ en el tercio proximal y el $30 \%$ restante en el hilio y en la misma glándula $(8,12)$. En este estudio, en 17 pacientes $(25,4 \%)$ se palpó un cálculo en la exploración y en todos los casos fue en el conducto de Wharton, $47,01 \%$ en la porción más proximal/hilio de la glándula y $52,99 \%$ en los dos tercios más distales del conducto, resultados muy similares a los descritos en la literatura (12).

La introducción de la sialoendoscopia ha significado un cambio en la visión del cirujano a la hora de plantear un tratamiento, y actualmente el interés se centra en realizar técnicas mínimamente invasivas (13). La evaluación diagnóstica preoperatoria es esencial para minimizar el riesgo de fracasos (14) y de ahí la importancia de solicitarlas de manera sistemática en nuestro centro, observando como la TC nos permite valorar de manera precisa la patología litiásica y la $\mathrm{RM}$, sobre todo si hablamos de la sialo-RM en la patología parotídea; centros con mucha experiencia en sialo-RM prefieren esta última.

Desde la incorporación de la técnica de sialoendoscopia en nuestro centro, la actitud ante la patología salival y su tratamiento ha cambiado considerablemente. En nuestra serie, 10 pacientes presentaban una litiasis palpable a nivel del conducto de Wharton. En los casos de litiasis de un tamaño $>8 \mathrm{~mm}$ y localizadas en la porción proximal del Wharton se realizó una resección transoral de la litiasis con el apoyo del sialoendoscopio y posterior exploración de los conductos. Únicamente en un paciente se realizó una submaxilectomía por presentar una litiasis intraglandular de $1,5 \mathrm{~cm}$. Aquellos pacientes con litiasis menores de $4 \mathrm{~mm}$ y con indicación de sialoendoscopia por número de episodios (cuatro pacientes) están a la espera de la realización de una sialoendoscopia básica y extracción de la litiasis. De los pacientes con litiasis parotídea (dos pacientes), ninguno presentó la litiasis en el tercio distal del conducto de Stenon. En todos los casos el tamaño fue $>4 \mathrm{~mm}$, y los pacientes optaron por un tratamiento conservador; estos resultados y el manejo fue similar a los hallados en la revisión sistemática de Atienza y colaboradores $(13,15,16)$.

La confirmación de que podemos resolver la enfermedad sin resecar la glándula debe animarnos a retomar los abordajes transorales descritos hace años y realizarlos en aquellos casos en que el volumen de las litiasis no permite la extracción por técnicas intraductales puras, o en aquellos casos de litiasis palpables en suelo de boca, aunque no se disponga del sialoendoscopio. La experiencia y los resultados de muchas series $(13,14,17-19)$ demuestran las ventajas del enfoque transoral tanto desde el punto de vista del posoperatorio (menor dolor y menor número de complicaciones) como de la posibilidad de volver a la actividad regular en un período más corto. De esta manera, también es posible conservar la glándula y su función $(20,21)$.
En cuanto a la patología no litiásica, solo dos pacientes de nuestra serie presentaron antecedentes de tratamiento con yodo radiactivo por un carcinoma papilar de tiroides. La sialoendoscopia seguida del lavado y tratamiento con corticoesteroides intraductales ofrece muy buenos resultados, ya que disminuye el número de episodios de sialoadenitis (9). Según Bulut y colaboradores, los pacientes con xerostomía secundaria al tratamiento con yodo radiactivo tratados con sialoendoscopia presentaban una mejora en la puntuación del cuestionario de xerostomía comparado con aquellos que optan por la abstención terapéutica $(22,23)$.

Los beneficios de la sialoendoscopia también han sido ampliamente estudiados en la parotiditis recurrente juvenil, cuando los episodios son muy recurrentes y con clínica severa (18). En nuestra serie estudiamos tres pacientes pediátricos con esta patología. aunque en ningún caso se realizó sialoendoscopia debido a su evolución favorable.

En cuanto a los pacientes con patología autoinmune (síndrome de Sjögren, síndrome seco secundario y parotiditis crónica secundaria a lupus eritematoso sistémico), en los que se planteó la realización de sialoendoscopia coincidiendo con la literatura, los hallazgos más frecuentes fueron estenosis y tapones mucosos, depósitos fibrinosos, palidez mucosa y ausencia de vascularización. Según Atienza y colaboradores, la tasa de éxito de la sialoendoscopia en los pacientes con patología autoinmune se sitúa en el 71 \% (13).

\section{Conclusión}

La patología obstructiva crónica benigna de las glándulas salivales tiene una alta prevalencia en la población general; por ello, es importante conocerla y tratarla correctamente. Realizar un diagnóstico correcto tanto clínico como radiológico es fundamental para planificar el mejor tratamiento. Aunque la TC tiene una alta sensibilidad para el diagnóstico de litiasis, la sialo-RM presenta resultados prometedores. Con la incorporación de técnicas mínimamente invasivas, como los abordajes transorales y la sialoendoscopia, el manejo de esta patología ha cambiado en los últimos años y limitado en gran medida la realización de resecciones glandulares.

La principal limitación de nuestro estudio radica en que se trata de una muestra limitada (67 pacientes, 17 tratados con ayuda del sialoendoscopio). Por otro lado, la sialoendoscopia requiere una curva de aprendizaje y, aunque los resultados son muy reveladores, debido a nuestra corta experiencia solo podemos hablar de un resultado preliminar y fundamentalmente en un cambio a la hora de abordar esta patología. Es necesario ampliar el estudio con un mayor número de pacientes, así como su seguimiento a largo plazo. La realización de la sialoendoscopia mejora con el tiempo y la experiencia, dependiendo de la curva de aprendizaje del cirujano $(14,24)$.

\section{Conflicto de intereses}

Los participantes en este estudio no tienen ningún conflicto de intereses. 


\section{Financiación}

Este estudio no está financiado parcial ni totalmente.

\section{Comité de ética}

La realización de este estudio fue aprobada por el Comité de ética de la Investigación Clínica (CEIC) de nuestro centro.

\section{REFERENCIAS}

1. Lee LI, Pawar RR, Whitley S, Makdissi J. Incidence of different causes of benign obstruction of the salivary glands: retrospective analysis of 493 cases using fluoroscopy and digital subtraction sialography. Br J Oral Maxillofac Surg. 2015 Jan;53(1):54-7. doi: 10.1016/j.bjoms.2014.09.017

2. Iro H, Zenk J, Escudier MP, Nahlieli O, Capaccio P, Katz P, et al. Outcome of minimally invasive management of salivary calculi in 4,691 patients. Laryngoscope. 2009;119(2):263-8. doi: 10.1002/lary.20008

3. Rasmussen ER, Lykke E, Wagner N, Nielsen T, Waersted S, Arndal H. The introduction of sialendoscopy has significantly contributed to a decreased number of excised salivary glands in Denmark. Eur Arch Otorhinolaryngol. 2016;273(8):2223-30. doi: 10.1007/s00405-015-3755-x

4. Nahlieli O. Endoscopic surgery of the salivary glands. Alpha Omegan. 2009;102(2):55-60. doi: 10.1016/j.aodf.2009.04.010

5. Zenk J, Koch M, Klintworth N, König B, Konz K, Gillespie MB, et al. Sialendoscopy in the diagnosis and treatment of sialolithiasis: a study on more than 1000 patients. Otolaryngol Head Neck Surg. 2012;147(5):858-63. doi: 10.1177/0194599812452837

6. Rzymska-Grala I, Stopa Z, Grala B, Golebiowski M, Wanyura H, Zuchowska A, et al. Salivary gland calculi - contemporary methods of imaging. Pol J Radiol. 2010;75(3):25-37.

7. Kopec T, Wierzbicka M, Szyfter W, Leszczynska M. Algorithm changes in treatment of submandibular gland sialolithiasis. Eur Arch Otorhinolaryngol. 2013;270(7):2089-93. doi: 10.1007/ s00405-013-2463-7

8. Lustmann J, Regev E, Melamed Y. Sialolithiasis. A survey on 245 patients and a review of the literature. Int J Oral Maxillofac Surg. 1990;19(3):135-8. doi: 10.1016/s0901-5027(05)80127-4

9. Harrison JD. Causes, natural history, and incidence of salivary stones and obstructions. Otolaryngol Clin North Am. 2009;42(6):927-47, Table of Contents. doi: 10.1016/j. otc. 2009.08.012

10. Ngu RK, Brown JE, Whaites EJ, Drage NA, Ng SY, Makdissi J. Salivary duct strictures: nature and incidence in benign salivary obstruction. Dentomaxillofac Radiol. 2007;36(2):63-7. doi: $10.1259 / \mathrm{dmfr} / 24118767$

11. Steck JH, Bertelli HD, Hoeppner CA, Volpi E, Vasconcelos EC. What Is the Learning Curve of Sialoendoscopy? Otolaryngology-Head and Neck Surgery. 2013;149(2_ suppl):P81-P81. doi: 10.1177/0194599813495815a143

12. Trujillo O, Rahmati RW. Acute and chronic salivary infection. En: Gland- Preserving Salivary Surgery: A Problem-Based Approach. Springer International Publishing; 2018. pp. 109-18.

13. Atienza G, López-Cedrún JL. Management of obstructive salivary disorders by sialendoscopy: a systematic review. $\mathrm{Br}$ J Oral Maxillofac Surg. 2015;53(6):507-19. doi: 10.1016/j. bjoms.2015.02.024

14. Gallo A, Capaccio P, Benazzo M, De Campora L, De
Vincentiis M, Farneti PA, et al. Risultati della scialoendoscopia interventistica nelle patologie ostruttive delle ghiandole salivari: Uno studio multicentrico Italiano. Acta Otorhinolaryngologica Italica. 2016;36(6):479-85. doi: 10.14639/0392-100X-1221

15. Capaccio P, Torretta S, Ottavian F, Sambataro G, Pignataro L. Modern management of obstructive salivary diseases. Acta Otorhinolaryngol Ital. 2007 Aug;27(4):161-72.

16. Nahlieli O, Nakar LH, Nazarian Y, Turner MD. Sialoendoscopy: A new approach to salivary gland obstructive pathology. J Am Dent Assoc. 2006 Oct;137(10):1394-400. doi: 10.14219/jada. archive.2006.0051

17. Kiringoda R, Eisele DW, Chang JL. A comparison of parotid imaging characteristics and sialendoscopic findings in obstructive salivary disorders. Laryngoscope. 2014;124(12):2696-701. doi: 10.1002/lary.24787

18. Francis CL, Larsen CG. Pediatric sialadenitis. Otolaryngol Clin North Am. 2014;47(5):763-78. doi: 10.1016/j.otc.2014.06.009

19. Saga-Gutiérrez C, Chiesa-Estomba CM, Larruscain E, González-García JÁ, Sistiaga JA, Altuna X. Transoral Sialolitectomy as an Alternative to Submaxilectomy in the Treatment of Submaxillary Sialolithiasis. Ear, Nose \& Throat Journal. 2019;98(5):287-90. doi:10.1177/0145561319841268

20. Marchal F, Kurt AM, Dulguerov P, Becker M, Oedman M, Lehmann W. Histopathology of submandibular glands removed for sialolithiasis. Ann Otol Rhinol Laryngol. 2001;110(5 Pt 1):464-9. doi: 10.1177/000348940111000513

21. Osailan SM, Proctor GB, Carpenter GH, Paterson KL, McGurk M. Recovery of rat submandibular salivary gland function following removal of obstruction: a sialometrical and sialochemical study. Int J Exp Pathol. 2006;87(6):411-23. doi: 10.1111/j.1365-2613.2006.00500.x

22. BulutOC, HaufeS, Hohenberger R, Hein M, KratochwilC, Rathke $\mathrm{H}$, et al. Impact of sialendoscopy on improving health related quality of life in patients suffering from radioiodineinduced xerostomia. Nuklearmedizin. 2018;57(4):160-167. English. doi: 10.3413/Nukmed-0964-18-03

23. Su YX, Xu JH, Liao GQ, Zheng GS, Cheng MH, Han L, Shan $\mathrm{H}$. Salivary gland functional recovery after sialendoscopy. Laryngoscope. 2009;119(4):646-52. doi: 10.1002/lary.20128

24. Farneti P, Macrì G, Gramellini G, Ghirelli M, Tesei F, Pasquini E. Learning curve in diagnostic and interventional sialendoscopy for obstructive salivary diseases. Acta Otorhinolaryngol Ital. 2015;35(5):325-31. doi: 10.14639/0392-100X-352 